\title{
Article \\ Pre-Operative Decitabine in Colon Cancer Patients: Analyses on WNT Target Methylation and Expression
}

\author{
Janneke F. Linnekamp ${ }^{1,2}$, Raju Kandimalla ${ }^{1}$, Evelyn Fessler ${ }^{1,+}$, Joan H. de Jong ${ }^{1,2}$, Hans M. Rodermond ${ }^{1,2}$, \\ Gregor G. W. van Bochove ${ }^{1,2} \mathbb{D}$, Frans O. The ${ }^{3} \mathbb{D}$, Cornelis J. A. Punt ${ }^{4}$, Willem A. Bemelman ${ }^{5}$, \\ Anthony W. H. van de Ven ${ }^{5,6}$, Pieter J. Tanis ${ }^{5}$ (D), Elles M. Kemper ${ }^{7}$, Lianne Koens ${ }^{8}$, Evelien Dekker ${ }^{9}$, \\ Louis Vermeulen ${ }^{1,2}$, Hanneke W. M. van Laarhoven ${ }^{4}$ and Jan Paul Medema ${ }^{1,2, *}$
}

1 Laboratory for Experimental Oncology and Radiobiology (LEXOR), Center for Experimental and Molecular Medicine, Amsterdam UMC, University of Amsterdam, Cancer Center Amsterdam, Meibergdreef 9, 1105 AZ Amsterdam, The Netherlands; j.f.linnekamp@amsterdamumc.nl (J.F.L.); rajbiochem@gmail.com (R.K.); fessler@genzentrum.lmu.de (E.F.); j.h.dejong@amsterdamumc.nl (J.H.d.J.); h.rodermond@amsterdamumc.nl (H.M.R.); g.g.vanbochove@amsterdamumc.nl (G.G.W.v.B.); l.vermeulen@amsterdamumc.nl (L.V.)

2 Oncode Institute, $1105 \mathrm{AZ}$ Amsterdam, The Netherlands

3 Department of Gastroenterology and Hepatology, OLVG, 1105 AZ Amsterdam, The Netherlands; fransolivier.the@usz.ch

check for updates

Citation: Linnekamp, J.F; Kandimalla, R.; Fessler, E.; de Jong, J.H.; Rodermond, H.M.; van Bochove, G.G.W.; The, F.O.; Punt, C.J.A.; Bemelman, W.A.; van de Ven, A.W.H.; et al. Pre-Operative Decitabine in Colon Cancer Patients: Analyses on WNT Target Methylation and Expression. Cancers 2021, 13, 2357. https://doi.org/10.3390/ cancers13102357

Academic Editors: Marta Baiocchi, Ann Zeuner and Isabelle Van Seuningen

Received: 31 January 2021

Accepted: 8 May 2021

Published: 13 May 2021

Publisher's Note: MDPI stays neutral with regard to jurisdictional claims in published maps and institutional affiliations.

Copyright: (c) 2021 by the authors. Licensee MDPI, Basel, Switzerland. This article is an open access article distributed under the terms and conditions of the Creative Commons Attribution (CC BY) license (https:/ / creativecommons.org/licenses/by/ $4.0 /)$.
4 Department of Medical Oncology, Cancer Center Amsterdam, Amsterdam UMC, University of Amsterdam, 1105 AZ Amsterdam, The Netherlands; c.j.a.punt@umcutrecht.nl (C.J.A.P.);

h.vanlaarhoven@amsterdamumc.nl (H.W.M.v.L.)

5 Department of Surgery, Amsterdam UMC, University of Amsterdam, Cancer Center Amsterdam, 1105 AZ Amsterdam, The Netherlands; w.a.bemelman@amsterdamumc.nl (W.A.B.); a.w.vandeven@amsterdamumc.nl (A.W.H.v.d.V.); p.j.tanis@amsterdamumc.nl (P.J.T.)

6 Department of Surgery, Flevo Hospital Almere, 1315 RA Almere, The Netherlands

7 Department of Pharmacology, Amsterdam UMC, University of Amsterdam, 1105 AZ Amsterdam, The Netherlands; e.m.kemper@amsterdamumc.nl

8 Department of Pathology, Amsterdam UMC, University of Amsterdam, 1105 AZ Amsterdam, The Netherlands; 1.koens@amsterdamumc.nl

9 Department of Gastroenterology and Hepatology, Amsterdam UMC, University of Amsterdam, Cancer Center Amsterdam, 1105 AZ Amsterdam, The Netherlands; e.dekker@amsterdamumc.nl

* Correspondence: j.p.medema@amsterdamumc.nl

+ Current address: Gene Center and Department of Biochemistry, Ludwig-Maximilians-Universität München, 80539 Munich, Germany.

Simple Summary: Colon cancer is one of the leading causes of cancer-related death worldwide. Therefore, the development of new therapeutic strategies is of the utmost importance. Previously, we identified a subset of colon cancers that are characterised by DNA methylation and have a poor prognosis. In this study, we therefore treated ten colon cancer patients with a demethylating agent, decitabine, to investigate if reversal of methylation is feasible and can be used as a novel therapy. Unfortunately, this study revealed that while decitabine treatment is effective in vitro, it only marginally decreased global methylation in patients and had no effect on the specific regions of DNA methylation in the tumours. Future studies should therefore focus on optimisation of treatment schedules in patients with highly methylated tumours.

Abstract: DNA hypermethylation is common in colon cancer. Previously, we have shown that methylation of WNT target genes predicts poor prognosis in stage II colon cancer. The primary objective of this study was to assess whether pre-operative treatment with decitabine can decrease methylation and increase the expression of WNT target genes APCDD1, AXIN2 and DKK1 in colon cancer patients. A clinical study was conducted, investigating these potential effects of decitabine in colon cancer patients (DECO). Patients were treated two times with $25 \mathrm{mg} / \mathrm{m}^{2}$ decitabine before surgery. Methylation and expression of LINE1 and WNT target genes (primary outcome) and expression of endogenous retroviral genes (secondary outcome) were analysed in pre- and post-treatment tumour samples using pyrosequencing and rt-PCR. Ten patients were treated with decitabine and eighteen patients were used as controls. Decitabine treatment only marginally decreased LINE1 
methylation. More importantly, no differences in methylation or expression of WNT target or endogenous retroviral genes were observed. Due to the lack of an effect on primary and secondary outcomes, the study was prematurely closed. In conclusion, pre-operative treatment with decitabine is safe, but with the current dosing, the primary objective, increased WNT target gene expression, cannot be achieved.

Keywords: decitabine; colon cancer; DNA methylation; clinical translation study

\section{Introduction}

The genetic aberrations in colon cancer have been extensively studied and are traditionally described by "the Vogelgram", starting with loss of functional APC, followed by mutations in other genes including KRAS, TP53 and SMAD4 [1]. In addition to genetic events, epigenetic alterations are frequently found and have been shown to be essential for the initiation and progression of colon cancer [2]. DNA methylation is associated with changes in the chromatin structure and results in altered gene expression without permanently changing the DNA sequence itself [3]. In various types of tumours, genomewide hypomethylation mainly occurs in repetitive sequences and can lead to genomic instability $[4,5]$. In contrast, DNA hypermethylation occurs in CpG islands in promotor regions of specific genes, resulting in transcriptional silencing (e.g., tumour suppressor genes), methylation of $C D K N 2 A$ in many cancers being an example [3,6,7]. Besides being an important step in tumourigenesis, DNA hypermethylation has also been suggested to cause resistance to systemic therapy $[8,9]$.

In colon cancer, relevant tumour suppressor genes are epigenetically silenced by DNA hypermethylation. For example, silencing of MLH1, a DNA mismatch repair gene, results in microsatellite instable (MSI) tumours. Methylation of MLH1 as well as other genes is encompassed in the $\mathrm{CpG}$ island Methylator Phenotype (CIMP). This phenotype is characterised by global hypermethylation and, in proximal tumours, is associated with worse prognosis $[10,11]$. Methylation of several other genes with biological, predictive or prognostic relevance has also been reported $[12,13]$. Previously, we have shown that methylation of the WNT target genes APCDD1, AXIN2 and DKK1 predicts poor prognosis in stage II colon cancer patients $[14,15]$. These genes can be methylated in both CIMP high, low and negative samples and are all negative regulators of the WNT pathway by negative feedback [16-18]. Therefore, inactivation of these genes by methylation can lead to activation of the WNT pathway. Importantly, even in APC mutant CRC, some level of WNT pathway regulation is still observed and inactivation of WNT pathway inhibitors is therefore thought to further tune the pathway.

DNA hypermethylation is facilitated by a group of enzymes called DNA methyltransferases (DNMTs) [19]. Azacitidine and decitabine are the best-known examples of DNMT inhibitors and are FDA-approved for myelodysplastic syndrome (MDS) and acute myeloid leukaemia $[20,21]$. In preclinical studies, we showed re-expression of WNT target genes in xenografted tumours after treatment of mice with the demethylating agent azacitidine [14]. Moreover, a subsequent decrease in tumour growth was observed [14]. These findings suggest that DNA methylation could be a therapeutic target in colon cancer and inducing re-expression could potentially lead to improved patient outcomes, especially in tumours characterised by extensive WNT target gene methylation.

The relationship between the clinical efficacy and the underlying molecular mechanisms of demethylating agents remains unclear, especially whether clinical response is a direct result of global demethylation [22,23]. The discrepancy between changes in methylation and clinical effect in several studies suggests that other factors in addition to methylation, such as immune regulation, are involved in patient response. One recent hypothesis is that endogenous retroviruses (ERV), integral parts of the human genome and silenced by methylation, are re-activated upon demethylation by DNMT inhibiting agents. 
This results in an interferon-like immune response in tumour cells, which finally leads to cell death $[23,24]$. Whether this indeed explains the therapeutic effect in patients needs to be further investigated. Facilitating this immune recognition and response could therefore be a promising new strategy.

The aim of this study was to examine the effect of decitabine in colon cancer. A translational clinical study was conducted, investigating the effect of pre-operative decitabine on the methylation and expression of WNT target genes APCDD1, AXIN2 and DKK1 and global methylation in colon cancer patients.

\section{Materials and Methods}

\subsection{Patient Recruitment and Inclusion Criteria}

The DECO study (NCT01882660) was conducted from February 2014 until December 2017. The study was approved by the Medical Ethical Committee of the Academic Medical Center (AMC), Amsterdam. Patients were approached in the outpatient clinic from the AMC and Onze Lieve Vrouwe Gasthuis (OLVG) in Amsterdam and Flevo Ziekenhuis in Almere, all in the Netherlands. Initial clinical staging was performed based on CT scan. Diagnosis was based on endoscopical view, CT scan and/or biopsies, and indication for tumour resection was determined by a multidisciplinary panel. Eligible participants included both male and female patients of 18 years or older, with colon cancer, who had an indication for primary tumour resection. Other inclusion criteria for decitabine treatment included: Karnofsky Performance Score $>70$, adequate bone marrow function and adequate hepatic and renal function. Finally, written informed consent had to be signed. Exclusion criteria included known hypersensitivity to decitabine or its additives or if surgery was not planned according to time frame of the study. Moreover, patients who received other systemic or local treatment of the primary tumour in the waiting time until surgery and administration of any experimental drug within 60 days prior to the first dose of decitabine were also excluded.

Pre-treatment samples were taken during endoscopy. For a detailed description of tumour samples, see Section 2.2. Ten \pm two days before surgery, patients were treated with decitabine (kindly donated by Janssen-Cilag, The Netherlands) as two one-hour infusions at a dose of $25 \mathrm{mg} / \mathrm{m}^{2}$ on two consecutive days. On the day of surgery, directly after resection, a second (post-treatment) sample was taken from the resected primary tumour. Furthermore, pathological staging was performed. Predefined primary endpoint was re-expression of WNT target genes (APCDD1, ASCL2, AXIN2 and DKK1) measured by quantitative real-time PCR (rt-PCR) in both pre-treatment samples taken during endoscopy and compared with post-treatment samples taken directly after resection. Secondary endpoints included global (LINE1) and WNT target gene methylation (APCDD1, ASCL2, $A X I N 2$ and $D K K 1)$ and proliferation assessed by immunohistochemistry in the described pre- and post-treatment tumour samples. During the study, we performed a separate validation study on prediction of prognosis of WNT target gene methylation and showed no additional value of ASCL2 in analyses [15]. Therefore, results of expression and methylation of ASCL2 were not included in this study.

To investigate if tumour material from the same patient collected with different procedures (endoscopy vs. resection) was comparable considering methylation, expression and proliferation, a non-treated control cohort was included. Inclusion criteria were patients with colon cancer, older than 18 years, with a Karnofsky performance score $>70$. Moreover, if an extra endoscopy procedure was performed, written informed consent was obtained.

\subsection{Patient Samples}

For pre-treatment samples, biopsies from endoscopy were used. Post-treatment samples were collected from resection specimens. In the decitabine-treated cohort, initially, only freshly frozen material was used. In order to obtain fresh-frozen pre-treatment biopsies, an extra endoscopy was performed before surgery. Due to the invasiveness of this 
procedure, we experienced a low inclusion rate. As a result, the protocol was amended after five patients were included. For the next five patients, FFPE material from a previously performed diagnostic endoscopy was used and compared with FFPE material from surgery.

For the control cohort, six patients with fresh-frozen material were enrolled before the amendment; however, three were excluded. Exclusion reasons were: neo-adjuvant treatment $(n=1)$, only tumour samples from endoscopy were freshly frozen $(n=1)$ and quality of material was insufficient $(n=1)$. We completed the control cohort with twelve colon cancer patients for whom FFPE material was stored. In total, 18 patients were enrolled for the control group, of which 15 could be evaluated for methylation and expression.

For fresh-frozen samples, tissues were immediately snap-frozen using liquid nitrogen and stored at $-80^{\circ} \mathrm{C}$. FFPE samples were incubated in $4 \%$ buffered formaldehyde for a maximum of $24 \mathrm{~h}$ and then transferred to $70 \%$ ethanol. Thereafter, samples were dehydrated through $80 \%, 90 \%, 96 \%$ and $100 \%$ of ethanol and finally in 1-butanol and paraffin. For all tumours, multiple (2-5) pre- and post-treatment biopsies were obtained and tumour percentage was determined by HE staining. Two biopsies from each sampling were used for the final analyses. For LINE1 methylation in treated patients, also a technical replicate was performed and results were averaged for final outcome. For all treated patients, MSI/MSS status, CIMP status and mutation of TP53, KRAS and BRAF were determined. Since the numbers of patients were low, no subgroup analysis could be performed.

Pre- and post-treatment blood samples were collected for haematological toxicity, and Common Terminology Criteria for Adverse Events were used to monitor other toxicities. Pre-treatment blood samples were taken at the time of diagnosis as standard of care and did not require an extra sample. Post-treatment blood samples were taken at the day of surgery.

\subsection{DNA/RNA Isolation}

Genomic DNA (gDNA) and RNA from the fresh-frozen patient samples were extracted with the AllPrep DNA/RNA/miRNA Universal Kit (Qiagen, Hilden, Germany) according to the manufacturer's instructions. For RNA, RNA integrity number values were determined using the Agilent 2100 bioanalyzer (see Table S1). FFPE tissue was cut into $10 \mu \mathrm{m}$ sections and deparaffinised. gDNA was isolated using a Nucleospin DNA FFPE xs kit (Machery-Nagel, Düren, Germany) following the manufacturer's instructions.

\subsection{Bisulfite Conversion and Pyrosequencing}

Bisulfite conversion was performed with 600-800 ng of gDNA using EZ DNA Methylation-Gold Kit (Zymo research, Irvine, CA, USA) according to the manufacturer's protocol. For the PCR of the bisulfite converted DNA (bcDNA), PyroMark PCR kit (Qiagen, Hilden, Germany) was used. In short, 20 ng of bcDNA was mixed with kit reagents and a subsequent amplification was performed on a thermocycler. Annealing temperatures were adjusted for different primers: for LINE1 and AXIN2, $56^{\circ} \mathrm{C}$ was used; for APCDD1, $58^{\circ} \mathrm{C}$; and for $D K K 1,52{ }^{\circ} \mathrm{C}$. Next, pyrosequencing was performed using $12 \mathrm{ng}$ of bcDNA. PyroMark Assay Design Software 2.0 (Qiagen, Hilden, Germany) was used for primer design and PCR and sequencing primers are listed in Table S2. For the WNT target genes, the exact location within the gene and CpG sites tested have been described before [15]. LINE1 sequence used was derived from Woloszynska-Read et al. [25]. The sequence analysed was 206-352 (Genbank accession number X52235.1) and contained three CpG sites. For validation, primers were also tested on DNA isolated from FFPE material and compared to DNA obtained from freshly frozen tissue before analysing patient material. This material originated from a previously conducted study, in which xenografts obtained from multiple colon cancer cell lines were used [26]. Results for the validation are shown in Figure S1 and show perfect correlation in methylation levels between the two distinct sample preparations. 


\subsection{Quantitative Real-Time PCR}

Complementary DNA (cDNA) was synthesised from $1 \mu \mathrm{g}$ of RNA using Superscript III reverse transcriptase (ThermoFisher, Waltham, MA, USA). For quantitative real-time $\mathrm{PCR}, 5 \mathrm{ng}$ of cDNA was used in a total reaction volume of $5 \mu \mathrm{l}$ containing $2.5 \mu \mathrm{L}$ of SYBR green and $0.5 \mu \mathrm{M}$ forward and reverse primer (see Table S3). Reaction was performed in a Lightcycler LC480 II (Roche).

\subsection{Ki67 Staining}

FFPE samples were used for Ki67 staining. Sections of a thickness of $4 \mu \mathrm{m}$ were prepared and deparaffinised using xylene and rehydrated through ethanol. Antigen retrieval was achieved using $10 \mathrm{mM}$ sodium citrate buffer $(\mathrm{pH}=6)$ (Vector Laboratories, Burlingame, CA, USA) for $20 \mathrm{~min}$ at $98{ }^{\circ} \mathrm{C}$. Samples were blocked using Dako REAL Peroxidase-Blocking Solution (Agilent technologies, Santa Clara, CA, USA) for $5 \mathrm{~min}$ at room temperature. Ki67 antibody (Sigma, SAB5500134, Saint Louis, MI, USA) was diluted 1:1000, in normal antibody diluent (Klinipath, ABB999, Duiven, The Netherlands), and incubated overnight at $4{ }^{\circ} \mathrm{C}$. After washing with PBS, poly HRP-anti Rabbit IgG (Bright vision, DPVR-55HRP, Immunologic, Duiven, The Netherlands) was added for $30 \mathrm{~min}$ at room temperature and finally stained using Bright DAB solution (3,3' diaminobenzidine, Immunologic, Duiven, The Netherlands). Counterstaining with haemotoxylin (Klinipath, 4085-9002, Duiven, The Netherlands) was incubated for $1 \mathrm{~min}$. After dehydration, slides were mounted using Pertex (HistoLab, Västra Frölunda, Sweden). For material from one patient (patient 9), no staining could be performed due to low quality of the material. For quantification of stainings, haemotoxylin colour was separated from DAB using the plugin "color deconvolution" in ImageJ. Positive nuclei were calculated as a percentage of total nuclei.

\subsection{CIMP Analysis}

For CIMP analyses, a panel of eight genes was used containing CACNA1G, CDKN2A, CRABP1, IGF2, MLH1, NEUROG1, RUNX3 and SOCS1. Furthermore, ALU was used to normalise for the amount of input bcDNA. Methods, primers and probes have been described previously [26,27]. Percentage of methylated reference (PMR) $>10$ was considered as positive. Tumours where $1-5$ out of 8 CIMP markers had a PMR $>10$ were defined as CIMP low. Tumours that had $\geq 6$ out of 8 markers with a PMR $>10$ were defined as CIMP high. Tumours were considered CIMP negative if none of the markers had a PMR $>10$.

\subsection{MSI/MSS}

Microsatellite stability was tested during standard of clinical care in the pathology department of our institute using immunohistochemistry for MLH1, MSH2, MSH6 and PMS2. If no results were available, a PCR-based MSI Analysis System, version 1.2 (Promega, Leiden, The Netherlands), was used. In this assay, the markers NR-21, BAT-26, BAT-25, NR-24, MONO-27, Penta C and Penta D were used. Assays were performed according to the manufacturers' instructions. Samples were considered as microsatellite instable if no staining was present in one of the four immunohistochemical stainings or more than 2 out of 5 markers of the PCR based analyses were instable.

\subsection{Mutational Status}

For mutational status, we used tumour samples obtained from resection. KRAS exon 2 and 3 and TP53 exon 1-11 were amplified by PCR, using 20 ng of gDNA (KRAS) or cDNA (TP53), $12.5 \mu \mathrm{L}$ Reddymix (ThermoFisher scientific), $1 \mu \mathrm{L}$ forward primer and reverse primer $10 \mu \mathrm{M}$ and $8.5 \mu \mathrm{L} \mathrm{H} \mathrm{H}_{2} \mathrm{O}$ in a total volume of $25 \mu \mathrm{L}$. For $K R A S$, thermocycler program was as follows: $5 \mathrm{~min} 95^{\circ} \mathrm{C}, 40$ cycles of $30 \mathrm{~s} 95^{\circ} \mathrm{C}, 30 \mathrm{~s} 50^{\circ} \mathrm{C}, 1 \mathrm{~min} 30 \mathrm{~s} 72^{\circ} \mathrm{C}$, followed by $5 \min 72^{\circ} \mathrm{C}$. We used the same protocol for TP53 only with an annealing temperature of $60^{\circ} \mathrm{C}$. Then, $0.1 \mu \mathrm{L}$ of the PCR product was sequenced by Big Dye Terminator 1.1 and subsequently analysed by direct Sanger sequencing. Primers are listed in Table S4. BRAF 
mutation was tested via quantitative rt-PCR with a wild type and BRAF V600E specific primer (Table S4). Reaction was performed using SYBR green by Lightcycler 480 . Ct value from $B R A F$ mutant was subtracted with $C t$ value of $B R A F$ wild type. Samples with differences of $<4 \mathrm{Ct}$ values were considered as $B R A F$ mutant.

\subsection{Statistical Analyses}

The planned maximum sample size for the decitabine treatment group as well as the control cohort was 44 with a $10 \%$ loss due to insufficient quality of material. We aimed to include twenty patients with high methylation of WNT target genes and twenty with lowly methylated WNT target genes. The group size was determined based on the incidence of methylation and the expected effect size. We expected a quarter of the tumours to be highly methylated for at least one gene based on results from a previous study [15]. The first interim analysis was performed after ten patients were included and treated with decitabine. For statistical analyses, GraphPad Prism 7 was used. To study the biological effect of decitabine in the clinical samples, we used a paired $t$-test to evaluate significant differences between pre- and post-treatment samples. For comparing the results of the Ki67 staining, a paired $t$-test was used. For all statistical comparisons, the level of significance was set at $p<0.05$.

\section{Results}

\subsection{Patient Characteristics}

To determine whether decitabine could decrease methylation and thereby re-express WNT target genes, a clinical trial (DECO) was conducted between February 2014 and December 2017. A total of ten colon cancer patients, nine male and one female, were enrolled and pre-operatively treated with decitabine, after which we performed an interim analysis that is reported here. Baseline characteristics and flow chart for inclusion are presented in Figure 1.
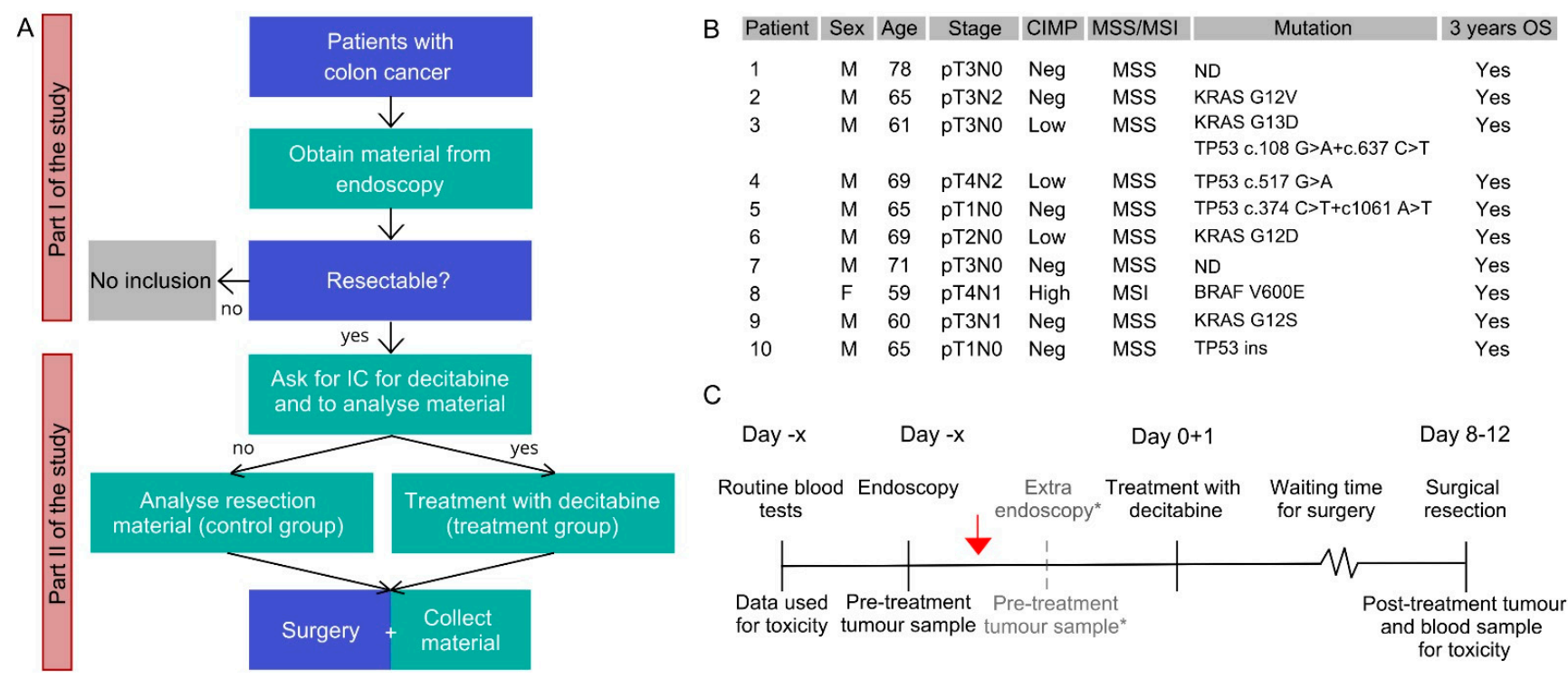

Figure 1. (A) Flow chart of DECO study. In blue squares, daily routine steps in clinical care are shown; in green squares, additional steps for DECO study are shown; (B) Baseline characteristics of decitabine-treated patients included in DECO study. Indicated stage is pathological staging after resection was done. Initial staging (for inclusion) was performed based on CT scan; (C) Timeline for DECO study. Red arrow shows the moment of inclusion. Blood tests before inclusion were part of standard of care. * For the patients with fresh frozen material, an extra endoscopy was performed to obtain freshly frozen biopsies as pre-treatment sample. For FFPE patients, pre-treatment samples were obtained from the diagnostic endoscopy performed for clinical purposes. IC = informed consent, $\mathrm{ND}=$ not detected, $\mathrm{OS}=$ overall survival. 
The median age was 65 years (range 59-78) and all patients were evaluable for toxicity, and, from all patients, material was available for methylation analyses. Furthermore, a nondecitabine-treated control cohort $(n=18)$ was enrolled, of which 15 patients were eligible for analyses. Although clinical outcome was not an endpoint of this study, three-year overall survival was documented and all patients reached this endpoint. In the follow-up after four years, two events occurred.

\subsection{Effect of Pre-Operative Treatment with Decitabine on Methylation in Colon Cancer Patients}

Before performing analyses on our primary endpoint, we verified if biopsies had a comparable percentage of methylation to tumour samples from resection using a control cohort. In this cohort, a total of 18 patients were included, of which 15 patients were available for analyses. To determine levels of global methylation, LINE1 methylation was used as a surrogate marker [28]. In this cohort, the average of LINE1 methylation for biopsies was $69.0 \pm 6.1 \%$ and resection material was $69.0 \pm 4.3 \%(n=15)$. Paired analysis revealed that biopsies and resection material could be directly compared, showing no statistical differences in methylation $(p=0.9718)$ (Figure 2A). Next, LINE1 methylation was assessed in decitabine-treated patients $(n=10)$. The average LINE1 methylation from the ten patients before treatment, as analysed on the biopsy material, was $71.2 \pm 6.4 \%$, while after treatment, the average was numerically lower (67.2 $\pm 6.5 \%)$. Paired analysis of the patients indicated that all but one patient showed a decrease in methylation and that this was significant when analysing the group ( $p=0.0075)$ (Figure 2A). Nevertheless, this decrease was relatively small for all patients tested, indicating that decitabine could modulate LINE1 methylation, but with the dosing used, the impact was minimal. To determine the impact of decitabine on WNT target gene methylation, CpG methylation of $A P C D D 1, A X I N 2$ and $D K K 1$ was measured in the first five patients from which fresh-frozen samples were available. Importantly, analysis of five patient sample pairs showed similar WNT target CpG methylation in pre- and post-treatment samples (Figure 2B) and no clear decrease could be detected. However, firm conclusions cannot be drawn for these data due to low patient numbers and the fact that these patients did not display high WNT target methylation at the start of treatment.

\subsection{Effect of Pre-Operative Treatment with Decitabine on Gene Expression and Proliferation in Colon Cancer Patients}

Despite the fact that only a small difference in LINE1 methylation and no clear impact on WNT target methylation could be detected, differences in gene expression or cell biological features, such as cell proliferation, could potentially be orchestrated without overt changes in methylation. To this end, WNT target gene and LINE1 expression was first analysed with quantitative rt-PCR in the fresh-frozen samples $(n=5)$. This revealed that both LINE1 and WNT target gene expression were not significantly different between pre- and post-treatment tumour samples (Figure 2C). 

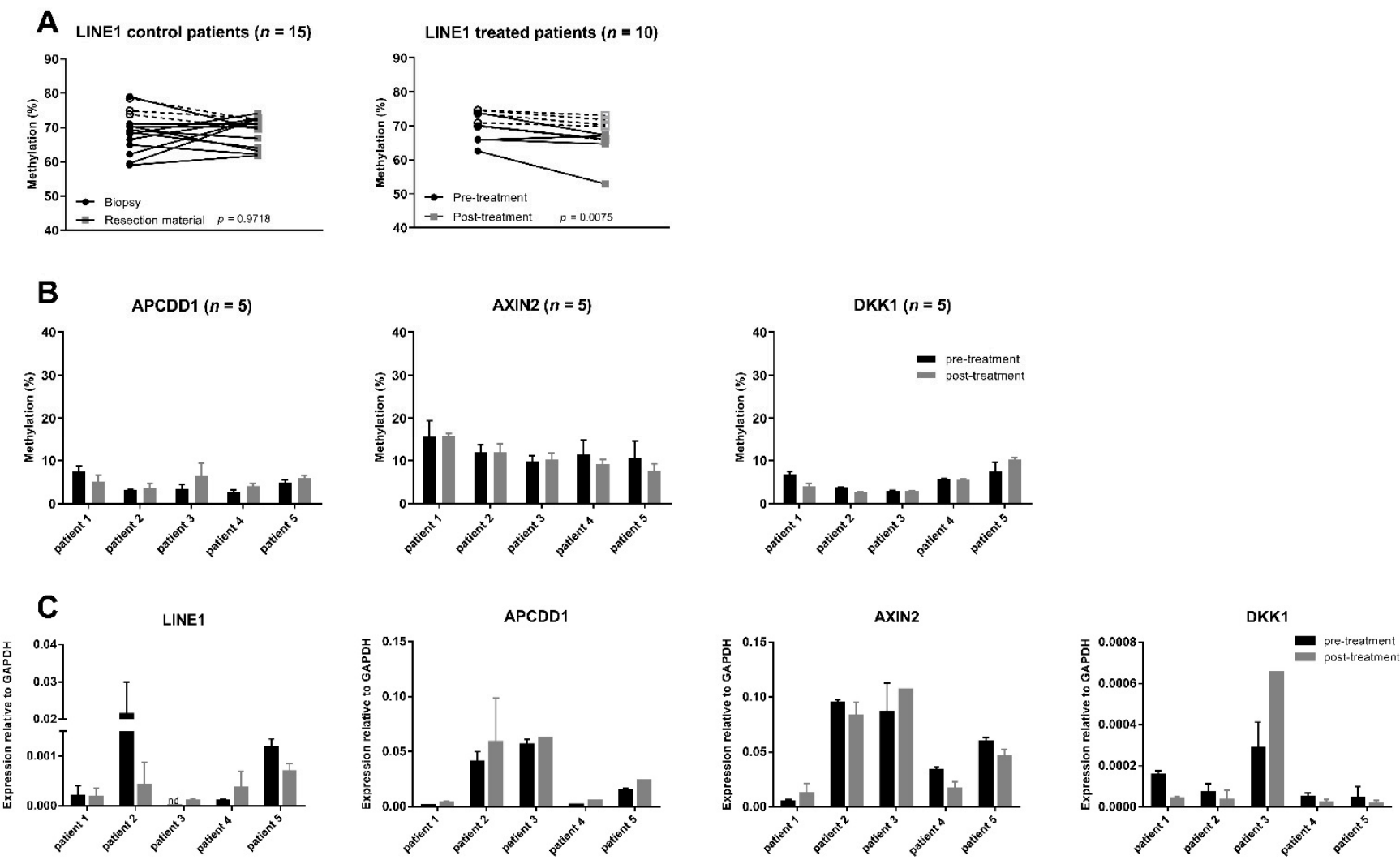

Figure 2. (A) Methylation of LINE1 in the control group $(n=15)$ and in the treated group $(n=10)$ (before and after treatment with $25 \mathrm{mg} / \mathrm{m}^{2}$ decitabine two times) measured by pyrosequencing. In both cohorts, FF tumour samples and FFPE samples were included. Open symbols and dotted lines represent FF samples, and closed symbols and lines represent FFPE samples. In the treated cohort, two technical replicates per time point were averaged and two biological replicates (two different samples from the same tumour) were used. For patient 6 to patient 10, no biological replicate was available for the pre-treatment sample. For statistical analyses, for pre-treatment and post-treatment samples, the average of all measurements was used. A paired $t$-test revealed no significant difference in the control cohort $(p=0.9718)$. For the treated cohort, a significant ( $p=0.0075)$ difference was shown; (B) Methylation of WNT target genes before and after treatment with decitabine in colon cancer patients measured by pyrosequencing $(n=5)$; (C) Expression of LINE1 and WNT target genes after treatment with decitabine measured by quantitative rt-PCR in fresh-frozen samples $(n=5)$. Values are the average of two samples (both for pre- and post-treatment samples), except for patient 3 , where only one post-treatment sample was available.

A reduction in proliferation after treatment with decitabine has been reported [29] and could also significantly impact the efficacy of demethylation as this is suggested to require cell cycle progression. Therefore, immunohistochemical staining for Ki67 was used to determine the expression on protein level (Figure 3). A large variation in Ki67 positive cells between patients as well as tumour region was observed, which is in line with earlier results [30]. However, no consistent difference between pre-treatment samples and post-treatment samples was detected (Figure 3B) $(p=0.7618)$. Although this may relate to the relatively small group size, we conclude that a strong impact on proliferation was not detected. This likely aligns with the lack of impact of a short course decitabine treatment on tumour growth. 
A
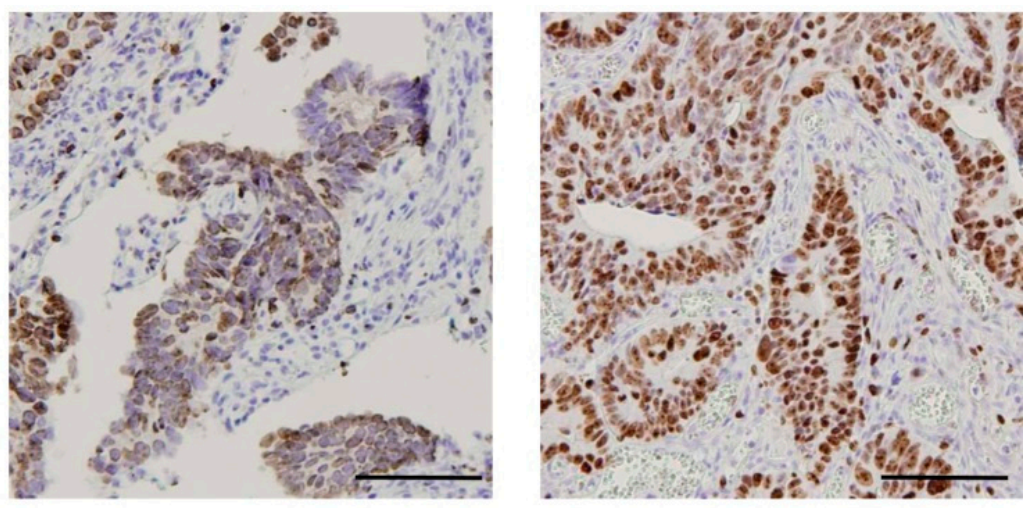

B

Ki67

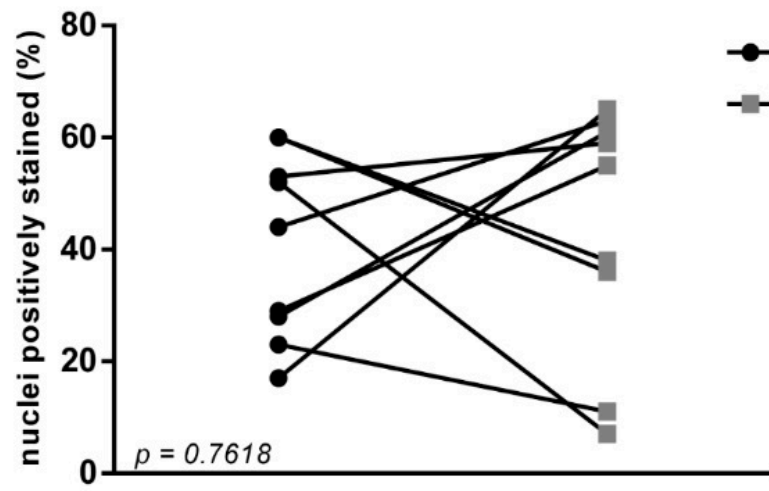

- pre-treatment

- post-treatment

Figure 3. (A) Representable Ki67 staining of biopsy and resection material of the tumour from one patient. The scale bar represents $100 \mu \mathrm{m}$; (B) Percentage of Ki67 positive cells compared to total cells from nine treated patients. A representative area of the tumour block was used for quantification. No significant difference between pre- and post-treatment samples $(p=0.7618)$ was observed using a paired $t$-test.

3.4. Decitabine Does Not Induce Expression of Endogenous Retrovirus ERVL and Interferon Associated Genes in Colon Cancer Patients

Endogenous retroviruses (ERVs) are heavily encoded in our human genome and effectively silenced by CpG methylation. Recent evidence suggests that decitabine can result in effective demethylation of these silencing $\mathrm{CpG}$ islands and result in reactivation of ERVs $[23,24]$. The cellular response towards ERV reactivation is rapid induction of interferon and interferon-related gene expression mounting an anti-viral and, as a result, anti-tumour immune response [23]. Importantly, as recent studies have also suggested that the inhibition of immune checkpoints in a neo-adjuvant setting is effective in colorectal cancer [31], we wondered whether decitabine could activate ERVs and hence provide an anti-tumour response. Therefore, the impact of decitabine on the gene expression of interferon-related genes and ERV ERVL was assessed in our patient tumour samples $(n=5$; DECO patient 1-5). However, although the patient numbers were limited, neither the reactivation of ERVL nor the activation of the interferon response was evident (Figure 4), suggesting that the levels of decitabine used in this study do not lead to ERV activation. 
ERVL

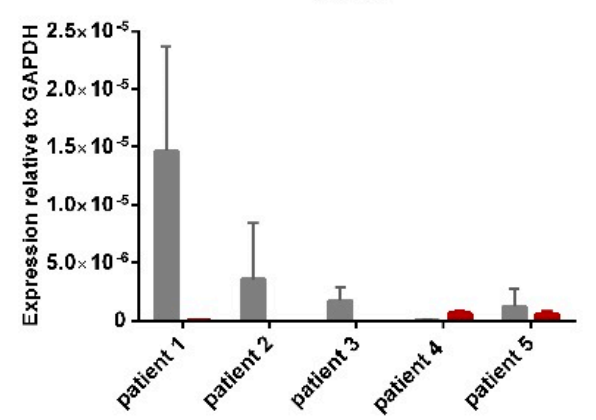

DDX58

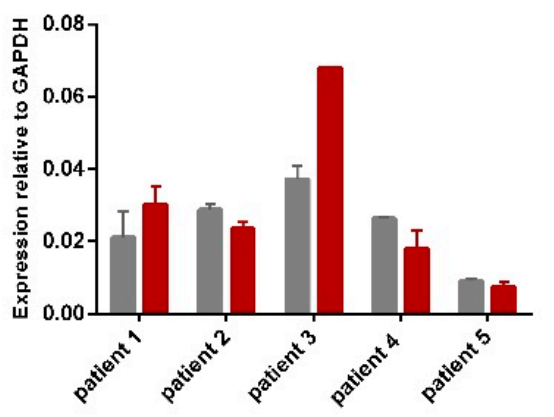

OASL

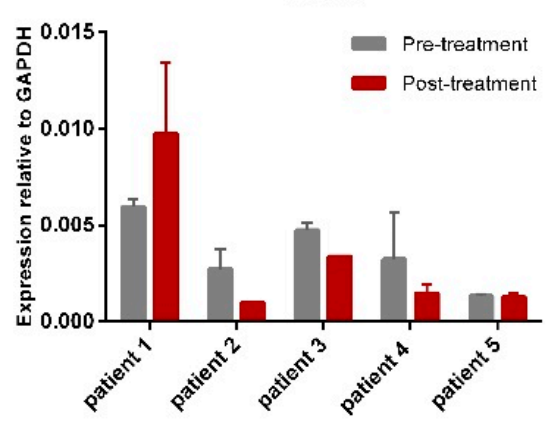

Figure 4. Expression of ERVL and interferon genes DDX58 and OASL in pre- and post-treatment samples $(n=2$ per patients except from patient 3) from patients treated with decitabine. Only fresh-frozen samples were used.

\subsection{Toxicity}

Grade 1 adverse events are summarised in Table S5 and pre- and post-treatment laboratory tests of the patients in Table S6. In addition, decitabine administration had no effect on the timing of surgery. This indicates that decitabine can be used safely preoperatively at the concentrations and timing used in this study.

\subsection{Study Closure}

Due to low patient inclusion, we amended the protocol after two years to use FFPE material to avoid the need for an extra endoscopy for patients. After including ten patients (not pre-specified) for the decitabine arm, the current analysis was performed. This revealed that decitabine treatment with the employed scheme did not result in demethylation and/or subsequent upregulation of WNT target genes partially controlled by methylation. The effect of decitabine on LINE1 methylation was significant, yet too limited to be impactful when analysing the expression of LINE1, while we also did not observe an impact on WNT target methylation or expression or on $E R V$ expression. Initially, we anticipated to include forty evaluable patients for decitabine treatment, aiming to change WNT target expression and methylation. However, with the results of the first ten patients, a different conclusion after forty patients was unlikely and we closed the study for further patient inclusion to avoid unnecessary impacts on patients.

\section{Discussion}

For early-stage colon cancer, surgery remains the cornerstone of treatment. However, in the case of stage III or high-risk stage II disease, adjuvant treatment with cytotoxic drugs improves patient outcomes. Although an overall survival benefit for cytotoxic treatment in these groups has been clearly documented, the proportion of patients with increased survival because of adjuvant therapy remains low. In clinical stage I-III colon cancer, neo-adjuvant therapy is currently not standard therapy, but a recent study shows that neo-adjuvant FOLFOX is safe, with no increase in perioperative morbidity [32]. This not only paves the way for studies assessing the long-term benefit of this strategy but also for the use of neo-adjuvant treatment for expeditious evaluation of response and thus for the development of new therapeutic options in colon cancer. CpG hypermethylation is an important event in tumourigenesis and its reversible nature makes it an attractive target for therapy. In addition to its role in tumour progression, CpG hypermethylation is associated with poor prognosis $[14,33,34]$, underlining the relevance of studying the effect of demethylating agents in colon cancer.

In this study, the biological effect of the demethylating drug, decitabine, was studied in colon cancer patients. A translational clinical study was conducted in which patients were treated with decitabine prior to surgery. The impact on LINE1 methylation using this treatment was significant but, compared to earlier studies, very small. In agreement, the observed decrease did not lead to an increase in LINE1 gene expression, nor did we observe 
a change in methylation of WNT target genes or WNT target gene or ERV expression. The minor effect on methylation was further corroborated by the observation that tumour proliferation did not change in the treated patients. In conclusion, this suggests that dosing decitabine twice at the concentration used, and after 8-12 days, is not sufficient to obtain impactful changes in tumour cells. It should be mentioned that the study was, due to pre-mature closure, underpowered. Furthermore, none of the patients analysed for WNT target gene methylation showed high levels of methylation for these genes and this could hamper the effect of decitabine. However, this level of methylation was not unexpected, as a previous study showed positive WNT target gene methylation in $26 \%$ of the tumours [15]. Nevertheless, the low methylation levels were also not affected by decitabine treatment, nor was the expression of the WNT target genes. In line with the limited effect on demethylation, a re-activation of endogenous retrovirus $E R V L$ as well as the linked interferon response was not observed in tumour material from a limited number of colon cancer patients treated with decitabine.

There are several explanations for the limited effect of decitabine in the patients in our study. Firstly, to prevent toxicity and, more importantly, an impact on the timing or success of the surgery, a relatively low dose of decitabine at only two injections was used, which could have resulted in a relatively low effective concentration in the patients. However, data from previous clinical studies suggest that the dose and timing that was used could be appropriate for demethylation in patients with solid tumours or myelodysplastic syndrome [35,36]. Nevertheless, repetitive administration and longer time intervals have been reported to optimise the effect [35,37-39]. Another explanation for our findings could be that decitabine is less effective in tumour tissue than in PBMCs, which are commonly used to monitor the effect on DNA methylation in patients [35,40,41]. Studies that compared the effect of methylation in PBMCs with tumour samples are limited and show conflicting results [35,42-45]. It is therefore difficult to extrapolate results from PBMCs to tumour samples as PBMCs are, by virtue of their location, more accessible for decitabine. PBMCs were not collected during our study as our focus was on the resected tumour tissue. However, for additional information about treatment schedules, collection of PBMCs might be useful in future studies. Moreover, collection of circulating tumour DNA could be insightful to evaluate response on methylation and prevent invasive biopsies [42,45-47]. Finally, a lower proliferation rate in tumour cells in patients compared to xenografts or in vitro cultures could also impair the effect of decitabine. Decitabine is only active during cell proliferation and demethylation is progressive with each cell division. Although the proliferation rate in these patient samples measured by Ki67 staining was relatively high, this still could be lower than in vitro. Nevertheless, no correlation between Ki67 staining in pre-treatment material and effect on LINE methylation was observed (data not shown).

The findings of a demethylating agent in colon cancer patients in this study are in line with several previously reported clinical studies in solid tumours [48]. Thus far, four studies on demethylating agents in colon cancer have been conducted. In a clinical trial using a combination of decitabine with panitumumab, a 10\% response rate was shown, but no effect on MAGE re-expression was observed [9]. In another study, capecitabine and oxaliplatin were combined with azacitidine in twenty-six colon cancer patients [46]. In this study, no objective response was observed, neither in CIMP high nor in CIMP low patients. In $60 \%$ of patients, methylation of vimentin was decreased; however, this effect was limited and did not outperform technical variation of methylation testing. More recently, guadecitabine was combined with irinotecan to treat metastatic colorectal cancer patients [37]. No consistent LINE1 demethylation was detected in tumour biopsies or circulating tumour DNA after 8 days. However, a reduction was seen after 15 days, although no correlation with clinical response could be observed [37]. This was in line with results from a study with 47 colon cancer patients, where demethylation was shown in post-treatment samples but was unrelated to response or overall survival [49]. Overall, the results of trials with demethylating agents in colon cancer are disappointing, although 
responses in individual patients are seen, emphasizing the importance of biomarkers to predict response or to find synergy with other drugs, e.g., immune checkpoint inhibitors.

Despite our findings, the study setup in which patients received neo-adjuvant treatment for a short period and tissue pre- and post-treatment was analysed is of interest for future drug studies. Our method facilitated the measurement of treatment response in colon cancer patients on primary tumour tissue on individual basis in a short time frame. Recent data on neo-adjuvant immunotherapy in colorectal cancer patients support this approach, and a more extensive analysis of the role of neo-adjuvant therapy in colon cancer is warranted. This study setup also allows for a quick evaluation of hypotheses and drugs that emanate from preclinical work. If an effect is detected, this strategy would allow for a rapid dissemination and identification of biomarkers to select patients for certain treatments. Thereby, this setup could potentially be used to personalise adjuvant treatment in colon cancer.

\section{Conclusions}

No decrease in WNT target gene methylation was observed after short-term preoperative treatment with decitabine in a limited amount of tumour tissue from five colon cancer patients. Future methylation studies should focus on optimisation of treatment regimens in patients with highly methylated tumours and perform parallel collection of PBMCs with tumour material.

Supplementary Materials: The following are available online at https:/ /www.mdpi.com/article/ 10.3390/cancers13102357/s1, Figure S1: Correlation between methylation of WNT target genes in DNA from fresh frozen (FF) and Formalin Fixed Paraffin Embedded (FFPE) samples from the same xenograft. All genes showed a high correlation, indicated with the correlation efficient $\mathrm{r} 2$ which was significant, Table S1: RIN values of samples used for quantitative real-time PCR. Table S2: Sequencing primers used for pyrosequencing, Table S3: Primers used for quantitative real-time PCR, Table S4: Primers used for mutation analyses, Table S5: Adverse events, Table S6: Laboratory test from preand posttreatment.

Author Contributions: Conceptualisation, J.F.L., F.O.T., C.J.A.P., A.W.H.v.d.V., P.J.T., E.M.K., E.D., L.V., H.W.M.v.L. and J.P.M.; Formal analysis, J.F.L., R.K. and J.P.M.; Funding acquisition, H.W.M.v.L. and J.P.M.; Investigation, J.F.L., R.K., J.H.d.J., H.M.R. and G.G.W.v.B.; Methodology, R.K., E.F. and J.P.M.; Project administration, J.F.L.; Resources, J.F.L., E.F., J.H.d.J., H.M.R., G.G.W.v.B., F.O.T., W.A.B., A.W.H.v.d.V., P.J.T., E.M.K., L.K. and E.D.; Supervision, E.D., L.V., H.W.M.v.L. and J.P.M.; Validation, L.K.; Visualisation, J.F.L.; Writing—original draft, J.F.L., H.W.M.v.L. and J.P.M.; Writing—review and editing, E.F., F.O.T., C.J.A.P., W.A.B., A.W.H.v.d.V., P.J.T., E.M.K., L.K., E.D. and L.V. All authors have read and agreed to the published version of the manuscript.

Funding: This research was funded by the KWF grant from the Dutch Cancer Society, grant number KWF 2012-542, and the Dutch Gastrointestinal and Liver disorder Foundation, grant number MLDS FP13-07, awarded to Jan Paul Medema. L.V. is a New York Stem Cell Foundation-Robertson Investigator.

Institutional Review Board Statement: The study was conducted according to the guidelines of the Declaration of Helsinki and approved by the Ethics Committee of the Academic Medical Center Amsterdam (2013_060, NL44048.018.13, Eudract number 2013-001060-38, 28 May 2013).

Informed Consent Statement: Informed consent was obtained from all subjects involved in the study.

Data Availability Statement: No new data were created or analyzed in this study. Data sharing is not applicable to this article.

Acknowledgments: We would like to thank M.W. Mundt for his help with patient inclusion. We would also like to thank A. Karpf for the help in sequence alignment. Decitabine was kindly donated by Janssen-Cilag.

Conflicts of Interest: The authors declare no conflict of interest. The funders had no role in the design of the study; in the collection, analyses, or interpretation of data; in the writing of the manuscript, or in the decision to publish the results. 


\section{References}

1. Fearon, E.R.; Vogelstein, B. A genetic model for colorectal tumorigenesis. Cell 1990, 61, 759-767. [CrossRef]

2. Okugawa, Y.; Grady, W.M.; Goel, A. Epigenetic Alterations in Colorectal Cancer: Emerging Biomarkers. Gastroenterology 2015, 149, 1204-1225.e12. [CrossRef]

3. Herman, J.G.; Baylin, S.B. Gene silencing in cancer in association with promoter hypermethylation. N. Engl. J. Med. 2003, 349, 2042-2054. [CrossRef]

4. Feinberg, A.P.; Vogelstein, B. Hypomethylation distinguishes genes of some human cancers from their normal counterparts. Nature 1983, 301, 89-92. [CrossRef]

5. Gama-Sosa, M.A.; Slagel, V.A.; Trewyn, R.W.; Oxenhandler, R.; Kuo, K.C.; Gehrke, C.W.; Ehrlich, M. The 5-methylcytosine content of DNA from human tumors. Nucleic Acids Res. 1983, 11, 6883-6894. [CrossRef]

6. Jones, P.A. Functions of DNA methylation: Islands, start sites, gene bodies and beyond. Nat. Rev. Genet. 2012, 13, 484-492. [CrossRef]

7. Herman, J.G.; Merlo, A.; Mao, L.I.; Lapidus, R.G.; Issa, J.P.J.; Davidson, N.E.; Sidransky, D.; Baylin, S.B. Inactivation of the CDKN2/p16/MTS1 gene is frequently associated with aberrant DNA methylation in all common human cancers. Cancer Res. 1995, 55, 4525-4530.

8. Glasspool, R.M.; Teodoridis, J.M.; Brown, R. Epigenetics as a mechanism driving polygenic clinical drug resistance. Br. J. Cancer 2006, 94, 1087-1092. [CrossRef]

9. Garrido-Laguna, I.; McGregor, K.A.; Wade, M.; Weis, J.; Gilcrease, W.; Burr, L.; Soldi, R.; Jakubowski, L.; Davidson, C.; Morrell, G.; et al. A phase I/II study of decitabine in combination with panitumumab in patients with wild-type (wt) KRAS metastatic colorectal cancer. Investig. New Drugs 2013, 31, 1257-1264. [CrossRef]

10. Ahn, J.B.; Chung, W.B.; Maeda, O.; Shin, S.J.; Kim, H.S.; Chung, H.C.; Kim, N.K.; Issa, J.-P.J. DNA methylation predicts recurrence from resected stage III proximal colon cancer. Cancer 2011, 117, 1847-1854. [CrossRef]

11. Juo, Y.Y.; Johnston, F.M.; Zhang, D.Y.; Juo, H.H.; Wang, H.; Pappou, E.P.; Yu, T.; Easwaran, H.; Baylin, S.; van Engeland, M.; et al. Prognostic value of $\mathrm{CpG}$ island methylator phenotype among colorectal cancer patients: A systematic review and meta-analysis. Ann. Oncol. 2014, 25, 2314-2327. [CrossRef] [PubMed]

12. Lam, K.; Pan, K.; Linnekamp, J.F.; Medema, J.P.; Kandimalla, R. DNA methylation based biomarkers in colorectal cancer: A systematic review. Biochim. Biophys. Acta 2016, 1866, 106-120. [CrossRef] [PubMed]

13. Draht, M.X.G.; Goudkade, D.; Koch, A.; Grabsch, H.I.; Weijenberg, M.P.; Van Engeland, M.; Melotte, V.; Smits, K.M. Prognostic DNA methylation markers for sporadic colorectal cancer: A systematic review. Clin. Epigenetics 2018, 10, 35. [CrossRef]

14. de Sousa, E.M.F.; Colak, S.; Buikhuisen, J.; Koster, J.; Cameron, K.; de Jong, J.H.; Tuynman, J.B.; Prasetyanti, P.R.; Fessler, E.; van den Bergh, S.P.; et al. Methylation of cancer-stem-cell-associated Wnt target genes predicts poor prognosis in colorectal cancer patients. Cell Stem Cell 2011, 9, 476-485.

15. Kandimalla, R.; Linnekamp, J.F.; Van Hooff, S.; Castells, A.; Llor, X.; Andreu, M.; Jover, R.; Goel, A.; Medema, J.P. Methylation of WNT target genes AXIN2 and DKK1 as robust biomarkers for recurrence prediction in stage II colon cancer. Oncogenesis 2017, 6, e308. [CrossRef]

16. Shimomura, Y.; Agalliu, D.; Vonica, A.; Luria, V.; Wajid, M.; Baumer, A.; Belli, S.; Petukhova, L.; Schinzel, A.; Brivanlou, A.H.; et al APCDD1 is a novel Wnt inhibitor mutated in hereditary hypotrichosis simplex. Nature 2010, 464, 1043-1047. [CrossRef]

17. Behrens, J.; Jerchow, B.-A.; Würtele, M.; Grimm, J.; Asbrand, C.; Wirtz, R.; Kühl, M.; Wedlich, D.; Birchmeier, W. Functional interaction of an axin homolog, conductin, with beta-catenin, APC, and GSK3beta. Science 1998, 280, 596-599. [CrossRef]

18. Zorn, A.M. Wnt signalling: Antagonistic Dickkopfs. Curr. Biol. 2001, 11, R592-R595. [CrossRef]

19. Okano, M.; Bell, D.W.; Haber, D.A.; Li, E. DNA methyltransferases Dnmt3a and Dnmt3b are essential for de novo methylation and mammalian development. Cell 1999, 99, 247-257. [CrossRef]

20. Gore, S.D.; Jones, C.; Kirkpatrick, P. Decitabine. Nat. Rev. Drug Discov. 2006, 5, 891-892. [CrossRef]

21. Issa, J.P.; Kantarjian, H.M.; Kirkpatrick, P. Azacitidine. Nat. Rev. Drug Discov. 2005, 4, 275-276. [CrossRef] [PubMed]

22. Issa, J.P.; Kantarjian, H.M. Targeting DNA methylation. Clin. Cancer Res. 2009, 15, 3938-3946. [CrossRef]

23. Roulois, D.; Yau, H.L.; Singhania, R.; Wang, Y.; Danesh, A.; Shen, S.Y.; Han, H.; Liang, G.; Jones, P.A.; Pugh, T.J.; et al. DNADemethylating Agents Target Colorectal Cancer Cells by Inducing Viral Mimicry by Endogenous Transcripts. Cell 2015, 162, 961-973. [CrossRef] [PubMed]

24. Chiappinelli, K.B.; Strissel, P.L.; Desrichard, A.; Li, H.; Henke, C.; Akman, B.; Hein, A.; Rote, N.S.; Cope, L.M.; Snyder, A.; et al. Inhibiting DNA Methylation Causes an Interferon Response in Cancer via dsRNA Including Endogenous Retroviruses. Cell 2015, 162, 974-986. [CrossRef]

25. Woloszynska-Read, A.; Mhawech-Fauceglia, P.; Yu, J.; Odunsi, K.; Karpf, A.R. Intertumor and intratumor NY-ESO-1 expression heterogeneity is associated with promoter-specific and global DNA methylation status in ovarian cancer. Clin. Cancer Res. 2008, 14, 3283-3290. [CrossRef] [PubMed]

26. Linnekamp, J.F.; Hooff, S.R.V.; Prasetyanti, P.R.; Kandimalla, R.; Buikhuisen, J.Y.; Fessler, E.; Ramesh, P.; Lee, K.; Bochove, G.G.W.; de Jong, J.H.; et al. Consensus molecular subtypes of colorectal cancer are recapitulated in in vitro and in vivo models. Cell Death Differ. 2018, 25, 616-633. [CrossRef] [PubMed] 
27. Ogino, S.; Kawasaki, T.; Kirkner, G.J.; Kraft, P.; Loda, M.; Fuchs, C.S. Evaluation of markers for CpG island methylator phenotype (CIMP) in colorectal cancer by a large population-based sample. J. Mol. Diagn. 2007, 9, 305-314. [CrossRef] [PubMed]

28. Yang, A.S.; Estecio, M.R.; Doshi, K.; Kondo, Y.; Tajara, E.H.; Issa, J.P. A simple method for estimating global DNA methylation using bisulfite PCR of repetitive DNA elements. Nucleic Acids Res. 2004, 32, e38. [CrossRef] [PubMed]

29. Stresemann, C.; Brueckner, B.; Musch, T.; Stopper, H.; Lyko, F. Functional diversity of DNA methyltransferase inhibitors in human cancer cell lines. Cancer Res. 2006, 66, 2794-2800. [CrossRef] [PubMed]

30. Prasetyanti, P.R.; van Hooff, S.R.; van Herwaarden, T.; de Vries, N.; Kalloe, K.; Rodermond, H.; van Leersum, R.; de Jong, J.H.; Franitza, M.; Nürnberg, P.; et al. Capturing colorectal cancer inter-tumor heterogeneity in patient-derived xenograft (PDX) models. Int. J. Cancer 2019, 144, 366-371. [CrossRef]

31. Chalabi, M.; Fanchi, L.F.; Dijkstra, K.K.; Berg, J.G.V.D.; Aalbers, A.G.; Sikorska, K.; Lopez-Yurda, M.; Grootscholten, C.; Beets, G.L.; Snaebjornsson, P.; et al. Neoadjuvant immunotherapy leads to pathological responses in MMR-proficient and MMR-deficient early-stage colon cancers. Nat. Med. 2020, 26, 566-576. [CrossRef]

32. Seymour, M.T.; Morton, D.; International FOxTROT Trial Investigators. FOxTROT: An international randomised controlled trial in 1052 patients (pts) evaluating neoadjuvant chemotherapy (NAC) for colon cancer. J. Clin. Oncol. 2019, 37 (Suppl. 15), 3504. [CrossRef]

33. Hiranuma, C.; Kawakami, K.; Oyama, K.; Ota, N.; Omura, K.; Watanabe, G. Hypermethylation of the MYOD1 gene is a novel prognostic factor in patients with colorectal cancer. Int. J. Mol. Med. 2004, 13, 413-417. [CrossRef]

34. Maeda, K.; Kawakami, K.; Ishida, Y.; Ishiguro, K.; Omura, K.; Watanabe, G. Hypermethylation of the CDKN2A gene in colorectal cancer is associated with shorter survival. Oncol. Rep. 2003, 10, 935-938. [CrossRef]

35. Appleton, K.; Mackay, H.J.; Judson, I.; Plumb, J.A.; McCormick, C.; Strathdee, G.; Lee, C.; Barrett, S.; Reade, S.; Jadayel, D.; et al. Phase I and pharmacodynamic trial of the DNA methyltransferase inhibitor decitabine and carboplatin in solid tumors. J. Clin. Oncol. 2007, 25, 4603-4609. [CrossRef]

36. Kantarjian, H.; Oki, Y.; Garcia-Manero, G.; Huang, X.; O’Brien, S.; Cortes, J.; Faderl, S.; Bueso-Ramos, C.; Ravandi, F.; Estrov, Z.; et al. Results of a randomized study of 3 schedules of low-dose decitabine in higher-risk myelodysplastic syndrome and chronic myelomonocytic leukemia. Blood 2007, 109, 52-57. [CrossRef]

37. Lee, V.; Wang, J.S.; Zahurak, M.L.; Gootjes, E.C.; Verheul, H.M.; Parkinson, R.M.; Kerner, Z.; Sharma, A.; Rosner, G.L.; De Jesus-Acosta, A.; et al. A Phase I Trial of a Guadecitabine (SGI-110) and Irinotecan in Metastatic Colorectal Cancer Patients Previously Exposed to Irinotecan. Clin. Cancer Res. 2018, 24, 6160-6167. [CrossRef]

38. Samlowski, W.E.; Leachman, S.A.; Wade, M.; Cassidy, P.; Porter-Gill, P.; Busby, L.; Wheeler, R.; Boucher, K.; Fitzpatrick, F.; Jones, D.A.; et al. Evaluation of a 7-day continuous intravenous infusion of decitabine: Inhibition of promoter-specific and global genomic DNA methylation. J. Clin. Oncol. 2005, 23, 3897-3905. [CrossRef]

39. Glasspool, R.M.; Brown, R.; Gore, M.E.; Rustin, G.J.S.; McNeish, I.A.; Wilson, R.H.; Pledge, S.; Paul, J.; MacKean, M.; Hall, G.D.; et al. A randomised, phase II trial of the DNA-hypomethylating agent 5-aza-2'-deoxycytidine (decitabine) in combination with carboplatin vs carboplatin alone in patients with recurrent, partially platinum-sensitive ovarian cancer. Br. J. Cancer 2014, 110, 1923-1929. [CrossRef]

40. Candelaria, M.; Gallardo-Rincón, D.; Arce, C.; Cetina, L.; Aguilar-Ponce, J.; Arrieta, Ó.; González-Fierro, A.; Chávez-Blanco, A.; de la Cruz-Hernández, E.; Camargo, M.; et al. A phase II study of epigenetic therapy with hydralazine and magnesium valproate to overcome chemotherapy resistance in refractory solid tumors. Ann. Oncol. 2007, 18, 1529-1538. [CrossRef]

41. Falchook, G.S.; Fu, S.; Naing, A.; Hong, D.S.; Hu, W.; Moulder, S.; Wheler, J.J.; Sood, A.K.; Bustinza-Linares, E.; Parkhurst, K.L.; et al. Methylation and histone deacetylase inhibition in combination with platinum treatment in patients with advanced malignancies. Investig. New Drugs 2013, 31, 1192-1200. [CrossRef]

42. Matei, D.; Fang, F.; Shen, C.; Schilder, J.; Arnold, A.; Zeng, Y.; Berry, W.A.; Huang, T.; Nephew, K.P. Epigenetic resensitization to platinum in ovarian cancer. Cancer Res. 2012, 72, 2197-2205. [CrossRef] [PubMed]

43. Zambrano, P.; Segura-Pacheco, B.; Perez-Cardenas, E.; Cetina, L.; Revilla-Vazquez, A.; Taja-Chayeb, L.; Chavez-Blanco, A.; Angeles, E.; Cabrera, G.; Sandoval, K.; et al. A phase I study of hydralazine to demethylate and reactivate the expression of tumor suppressor genes. BMC Cancer 2005, 5, 44. [CrossRef] [PubMed]

44. Aparicio, A.; Eads, C.A.; Leong, L.A.; Laird, P.W.; Newman, E.M.; Synold, T.W.; Baker, S.D.; Zhao, M.; Weber, J.S. Phase I trial of continuous infusion 5-aza-2'-deoxycytidine. Cancer Chemother. Pharmacol. 2003, 51, 231-239. [CrossRef] [PubMed]

45. Fang, F.; Balch, C.; Schilder, J.; Breen, T.; Zhang, S.; Shen, C.; Li, L.; Kulesavage, C.; Bs, A.J.S.; Nephew, K.P.; et al. A phase 1 and pharmacodynamic study of decitabine in combination with carboplatin in patients with recurrent, platinum-resistant, epithelial ovarian cancer. Cancer 2010, 116, 4043-4053. [CrossRef]

46. Overman, M.J.; Morris, V.; Moinova, H.; Manyam, G.; Ensor, J.; Lee, M.S.; Eng, C.; Kee, B.; Fogelman, D.; Shroff, R.T.; et al. Phase I/II study of azacitidine and capecitabine/oxaliplatin (CAPOX) in refractory CIMP-high metastatic colorectal cancer: Evaluation of circulating methylated vimentin. Oncotarget 2016, 7, 67495-67506. [CrossRef]

47. Juergens, R.A.; Wrangle, J.; Vendetti, F.P.; Murphy, S.C.; Zhao, M.; Coleman, B.; Sebree, R.; Rodgers, K.; Hooker, C.M.; Franco, N.; et al. Combination epigenetic therapy has efficacy in patients with refractory advanced non-small cell lung cancer. Cancer Discov. 2011, 1, 598-607. [CrossRef] 
48. Linnekamp, J.F.; Butter, R.; Spijker, R.; Medema, J.P.; van Laarhoven, H.W.M. Clinical and biological effects of demethylating agents on solid tumours-A systematic review. Cancer Treat. Rev. 2017, 54, 10-23. [CrossRef] [PubMed]

49. Azad, N.S.; El-Khoueiry, A.; Yin, J.; Oberg, A.L.; Flynn, P.; Adkins, D.; Sharma, A.; Weisenberger, D.J.; Brown, T.; Medvari, P.; et al. Combination epigenetic therapy in metastatic colorectal cancer (mCRC) with subcutaneous 5-azacitidine and entinostat: A phase 2 consortium/stand up 2 cancer study. Oncotarget 2017, 8, 35326-35338. [CrossRef] [PubMed] 